\title{
Оценка межмолекулярных взаимодействий трифенильных производных элементов пятой группы Периодической системы методом газо-жидкостной хроматографии
}

\author{
Танеева А.В., Ву Нгок Зан, Нгуен Зуи Хынг, \\ Дмитриева А.В., Новиков В.Ф.
}

Казанский государственный энергетический университет, Казань

Поступила в редакцию 25.03.2019 г.

DOI: $10.17308 /$ sorpchrom.2019.19/1171

Методом газо-жидкостной хроматографии изучили характер межмолекулярного взаимодействия трифенильных производных элементов пятой группы Периодической системы с органическими веществами различной физико-химической природы. Определены хроматографические факторы полярности Роршнайдера изученных рядов соединений, проведена оценка вклада в межмолекулярное взаимодействие полярных сорбатов на основе трехмерной модели и определены термодинамические функции сорбента. Показано влияние центрального атома молекулы трифенильных производных на их сорбционные свойства.

Ключевые слова: газо-жидкостная хроматография, сорбент, сорбат, органические производные, индексы удерживания, факторы полярности.

\section{Evaluation of intermolecular interactions of triphenyl derivatives of elements of the fifth group of the Periodic system by gas-liquid chromatography}

\author{
Taneeva A.V., Dmitrieva A.V., Vu Ngoc Zan, \\ Zuy Nguyen Hung, Novikov V.F.

\section{Kazan State Power Engineering University, Kazan}

There is carried out estimation of intermolecular interactions of triphenyl derivatives of elements of the fifth group of the Periodic system. The aim of this work is to identify main regularities of influence of the Central atom of an element on a chromatographic and thermodynamic properties triphenylene derivatives of the elements of the fifth group.

The method of work consists in the preparation of sorbents on the basis of triphenyl derivatives of the fifth group elements, loading them into the packing column and determining the chromatographic and thermodynamic properties of the obtained sorbents for the analysis of test substances, which were used as substances of different donor-acceptor nature. Determined their retention times on the basis of which was calculated chromatographic factors polarity of Rorshneider and enthalpy of sorption of these substances in triphenylene derivatives of the elements of the fifth group. There is determined the contribution to the retention of standard sorbates of different types of intermolecular interactions on the basis of the threedimensional model. It was relevated the character of intermolecular interactions of triphenyl derivatives of the fifth group elements with electron - donor and acceptor sorbates, was determined their enthalpy of sorption and it was found that in addition to phosphorus and arsenic compounds, promising materials for obtaining sorbents can be organic derivatives of bismuth and antimony. 
Thus, the method of gas-liquid chromatography shows the effect of the Central atom of the molecule triphenylene derivatives for their sorption properties in relation to organic substances. The results can be used in the synthesis of new sorbents for gas-liquid chromatography with controlled selectivity of organic matter separation by introducing various functional groups capable of intermolecular interaction into the structure of the molecule

Keywords: gas-liquid chromatography, sorbent, sorbate, organic derivatives, phosphorus, arsenic, nitrogen, bismuth, antimony, retention indices, polarity factors.

\section{Введение}

Как известно, метод газо-жидкостной хроматографии широко применяется в аналитической химии для контроля различных технологических процессов и определения концентрации органических и неорганических веществ, в том числе и примесных соединений. Сорбент, помещенный в хроматографическую колонку, может представлять самостоятельный научный интерес как объект исследования, в результате изучения которого представляется возможным производить оценку физикохимических свойств сорбентов на основании результатов анализа стандартных сорбатов различной природы. Ранее такая оценка была проведена нами с использованием некоторых классов органических производных мышьяка и фосфора [1-3].

Как известно, сорбенты, приготовленные на основе арсенированных производных, обладают более высокой гидроксильной селективностью перед известными аналогами, что определяется наличием неподеленной электронной пары арсенильного кислорода, способного к проявлению электронодонорных взаимодействий [4-5]. При этом установлено, что электронодонорные заместители в фенильном кольце молекулы алкиларсиновой кислоты оказывают существенное влияние на их селективные свойства [6].

Органические производные фосфора и мышьяка характеризуются некоторой общностью свойств, заключающейся в близкой электронодонорной способности. Фосфор и мышьяк относятся к пятой группе Периодической системы, в которую также входят азот, сурьма и висмут. Из литературных данных известно, что относительная электронодонорная способность химических соединений, образованных одной и той же группой Периодической системы, зависит от природы центрального атома и его окружения [7]. В случае трифенильных производных закономерности в изменении электронодонорной способности молекул существенно сложнее, поскольку эти соединения имеют различные электронодонорные центры [8].

В работе [9] на основе сопоставления экспериментально определенных молекулярных параметров, полученных с использованием различных физико-химических методов анализа, выведены корреляционные уравнения для рядов соединений элементов Пятой группы, на основе которых установлены общие закономерности, наблюдаемые при изменении природы заместителя и центрального атома в основном и возбужденном состоянии. Выявлены закономерности изменения дипольных моментов отдельных фрагментов молекул при изменении природы атомов элемента, что позволило оценить влияние заместителей на электронное строение центрального атома и рассчитать электрооптические параметры рассматриваемых молекул в возбужденном состоянии.

В продолжение этих исследований цель настоящей работы заключалась в оценке характера межмолекулярных взаимодействий трифенильных производных элементов пятой группы Периодической системы методом газо-жидкостной хроматографии. 


\section{Эксперимент}

Экспериментальную часть работы проводили на газо-жидкостном хроматографе марки Кристаллюкс-4000М с пламенно-ионизационным детектором и насадочными колонками из нержавеющей стали длиной 3м, внутренним диаметром 3 мм. Трифенильные производные элементов пятой группы Периодической системы были получены по методикам, опубликованным в литературе [10-11]. Органические жидкости, используемые в качестве неподвижных фаз для приготовления сорбентов, наносили на инертный твердый носитель марки Хроматон-N в количестве $15 \%$ от массы последнего. Определяли времена удерживания стандартных сорбатов, в качестве которых использовали предельные углеводороды, а также бензол, этанол, метилэтилкетон, нитрометан и пиридин, на основе которых рассчитывали их характеристики удерживания в соответствии с требованиями нормативной документации [1214]. На основании логарифмических индексов удерживания бензола, этанола, метилэтилкетона, нитрометана и пиридина рассчитывали хроматографические факторы полярности Роршнайдера, которые описывают способность сорбатов к донорноакцепторному и ориентационному взаимодействию в системе сорбат-сорбент [1617]. По результатам данных хроматографирования рассчитывали термодинамические характеристики изучаемых сорбентов [18]. Для оценки вклада в межмолекулярное взаимодействие полярных сорбатов использовали трехмерную модель (тетраэдр), заключающуюся в применении объемной треугольной системы координат [19].

\section{Обсуждение результатов}

Как известно, физико-химические свойства элементоорганических соединений наиболее ярко проявляются при их межмолекулярном взаимодействии с различными по природе веществами и зависят от природы центрального атома и его окружения. При этом увеличение размеров центрального атома элемента уменьшает степень связанности электронов внешнего слоя с ядром, что, как правило, приводит к более легкому их отрыву.

В табл.1 приведены физико-химические и хроматографические свойства трифенильных производных элементов пятой группы Периодической системы [20-21]. Как видно из табл., атомные радиусы центрального атома и атомная масса возрастают от азота к фосфору, мышьяку, сурьме и висмуту, а электроотрицательность уменьшается. Температуры плавления исследуемых соединений характеризуются самыми различными значениями. Для фосфора, мышьяка, сурьмы и висмута она не превышает температуру $100^{\circ} \mathrm{C}$.

При определении логарифмических индексов удерживания стандартных сорбатов на трифенильных производных элементов пятой группы они имеют самые разные значения. Для всех исследуемых соединений наиболее высокие значения логарифмических индексов удерживания наблюдаются для сильного донора электронов - пиридина, при этом они наиболее высоки для соединений висмута. Более низкие значения логарифмических индексов удерживания сорбатов наблюдаются для этанола.

Таким образом, трифенильные производные элементов пятой группы Периодической системы способны вступать с анализируемыми сорбатами как в электронодонорное, так и в электроно-акцепторное взаимодействия различной силы, что характеризуется уменьшением значений логарифмических индексов удерживания в ряду: фосфор $\rightarrow$ мышьяк $\rightarrow$ сурьма $\rightarrow$ висмут $\rightarrow$ азот, как для доноров (бензол, пиридин), так и акцепторов (нитрометан) электронов. 
Таблица 1. Физико-химические и хроматографические свойства трифенильных производных элементов пятой группы Периодической системы [21-22] $\left(\mathrm{C}_{6} \mathrm{H}_{5}\right)_{3} Э$, где Э=N, P, As, Sb, Bi; M - атомная масса; $\mathrm{A}_{\mathrm{a}}{ }^{0}$-атомные радиусы при степени окисления $=0 ; \mathrm{T}_{\text {пл }}{ }^{\circ} \mathrm{C}-$ температура плавления,${ }^{0} \mathrm{C} ; \mathrm{J}-$ логарифмические индексы удерживания сорбатов; (x,y,z,u,s) - хроматографические факторы полярности Роршнайдера

\begin{tabular}{|c|c|c|c|c|c|c|c|c|c|}
\hline \multirow{2}{*}{$\begin{array}{l}\text { № } \\
\Pi / \Pi\end{array}$} & \multicolumn{4}{|c|}{$\left(\mathrm{C}_{6} \mathrm{H}_{5}\right)_{3} \ni$} & \multicolumn{5}{|c|}{$\begin{array}{c}\text { Логарифмические индексы удерживания сорбатов (J) и } \\
\text { хроматографические факторы полярности }(\mathrm{x}, \mathrm{y}, \mathrm{z}, \mathrm{u}, \mathrm{s})\end{array}$} \\
\hline & $Э$ & M & $\mathrm{A}_{\mathrm{a}}^{0}$ & $\begin{array}{l}\mathrm{T}_{\text {пл }} \\
{ }^{\circ} \mathrm{C}\end{array}$ & $\begin{array}{l}\text { Бензол } \\
\text { (x) }\end{array}$ & $\begin{array}{l}\text { Этанол } \\
\text { (у) }\end{array}$ & $\begin{array}{l}\text { Метилэтил- } \\
\text { кетон }(\mathrm{z})\end{array}$ & $\begin{array}{c}\text { Нитро- } \\
\text { метан (u) }\end{array}$ & $\begin{array}{l}\text { Пиридин } \\
\text { (s) }\end{array}$ \\
\hline 1 & $\mathrm{~N}$ & 14 & 0.71 & 127 & $714 / 0.65$ & $652 / 2.95$ & $761 / 2.30$ & $741 / 2.81$ & $896 / 1.36$ \\
\hline 2 & $\mathrm{P}$ & 31 & 1.30 & 76 & $814 / 1.65$ & $671 / 2.74$ & $714 / 1.83$ & $769 / 3.09$ & $956 / 1.96$ \\
\hline 3 & As & 75 & 1.48 & 56 & $795 / 1.46$ & $548 / 3.03$ & $717 / 2.70$ & $730 / 2.70$ & $921 / 1.61$ \\
\hline 4 & $\mathrm{Sb}$ & 122 & 1.61 & 41 & $784 / 1.35$ & $660 / 2.18$ & $687 / 1.56$ & $736 / 2.76$ & $926 / 1.66$ \\
\hline 5 & $\mathrm{Bi}$ & 209 & 1.82 & 72 & $796 / 1.47$ & $575 / 2.18$ & $735 / 2.04$ & $773 / 3.13$ & $958 / 1.98$ \\
\hline
\end{tabular}

Для этанола, который способен к образованию водородной связи, характер приведенной зависимости изменяется и происходит в последовательности: фосфор $\rightarrow$ сурьма $\rightarrow$ азот $\rightarrow$ висмут $\rightarrow$ мышьяк.

Метилэтилкетон характеризуется более высокими значениями удерживания на сорбенте, приготовленном на основе трифенильных производных атома азота и располагается в ряд: азот $\rightarrow$ висмут $\rightarrow$ мышьяк $\rightarrow$ фосфор $\rightarrow$ сурьма.

Для акцептора электронов - нитрометана логарифмические индексы удерживания располагаются в ряд: висмут $\rightarrow$ фосфор $\rightarrow$ азот $\rightarrow$ сурьма $\rightarrow$ мышьяк.

Донор электронов - пиридин вступает в более сильное взаимодействие с висмутом и располагается в ряд: висмут $\rightarrow$ фосфор $\rightarrow$ сурьма $\rightarrow$ мышьяк $\rightarrow$ азот.

При этом для элементов пятой группы Периодической системы в случае полярных сорбатов наблюдается изменение логарифмических индексов удерживания в порядке повышения температур кипения анализируемых сорбатов: этанол $\left(T_{\text {кии }}=78^{\circ} \mathrm{C}\right) \rightarrow$ метилэтилкетон $\left(T_{\text {кии }}=80^{\circ} \mathrm{C}\right) \rightarrow$ бензол $\left(T_{\text {кии }}=80.1^{\circ} \mathrm{C}\right) \rightarrow$ нитрометан $\left(T_{\text {кип }}=101^{\circ} \mathrm{C}\right) \rightarrow$ пиридин $\left(T_{\text {кии }}=114^{\circ} \mathrm{C}\right)$.

На рис.1 приведена зависимость логарифма приведенного удерживаемого объема н-алканов от числа атомов углерода в их молекуле для сорбентов, приготовленных на основе трифенильных производных элементов пятой группы Периодической системы. Как видно из рис.1 эти зависимости являются линейными для всех исследуемых соединений, что указывает на линейность изотермы сорбции в процессах растворения н-алканов в трифенильных производных элементов Периодической системы.

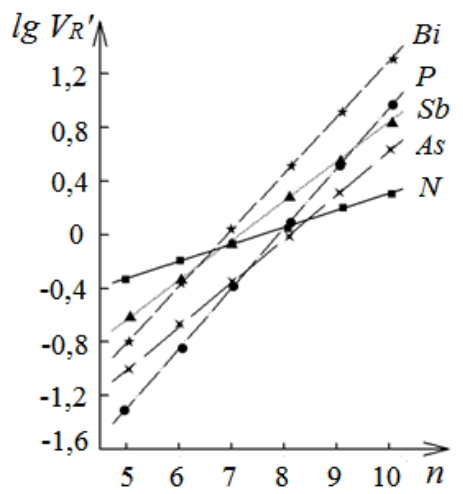

Рис. 1. Зависимости логарифма приведенного объема удерживания $\left(\lg V_{R}^{\prime}\right)$ н-алканов от числа атомов углерода в их молекуле (n) для элементов пятой группы Периодической системы ряда: $\left(\mathrm{C}_{6} \mathrm{P}_{5}\right)_{3} Э$; где: Э=As; $\mathrm{P} ; \mathrm{N} ; \mathrm{Sb} ; \mathrm{Bi}$ 
С целью энергетической оценки трифенильных производных элементов пятой группы Периодической системы проводилось измерение термодинамических параметров сорбции в них различных по физико-химической природе органических растворителей, экспериментальные значения которых приведены в таблице 2. Из табл. видно, что для сорбатов, способных к специфическому межмолекулярному взаимодействию, изменение мольной энтальпии сорбции имеет различные значения. В случае образования межмолекулярных водородных связей фосфорорганических соединений с этанолом наблюдаются экстремально высокие значения энтальпии сорбции $\left(-\Delta \mathrm{H}^{0}=41 \kappa\right.$ Дж/моль). Для трифенильных производных сурьмы, мышьяка и азота энтальпия сорбции имеет более низкие значения $\left(-\Delta \mathrm{H}^{0}=36,27\right.$ и 11 кДж/моль, соответственно).

Таблица 2. Изменение мольной энтальпии сорбции (- $\Delta \mathrm{H}^{\mathrm{o}}$, кДж/моль) стандартных сорбатов системы Роршнайдера в трифенильных производных элементов пятой группы Периодической системы ряда: $\left(\mathrm{C}_{6} \mathrm{H}_{5}\right)_{3}$ Э; где: Э=As ; P; N; $\mathrm{Sb} ; \mathrm{Bi}$

\begin{tabular}{|c|c|c|c|c|c|c|}
\hline \multirow{2}{*}{ №п/п } & \multirow{2}{*}{ Сорбат } & \multicolumn{5}{|c|}{$-\Delta \mathrm{H}^{0}$, кДж/моль } \\
\cline { 2 - 7 } & Пентан & $\mathrm{N}$ & $\mathrm{P}$ & $\mathrm{As}$ & $\mathrm{Sb}$ & $\mathrm{Bi}$ \\
\hline 1 & Гексан & 3 & 27 & 22 & 39 & 14 \\
\hline 2 & Гептан & 2 & 30 & 26 & 40 & 18 \\
\hline 3 & Октан & 2 & 32 & 29 & 41 & 22 \\
\hline 4 & Нонан & 3 & 37 & 32 & 41 & 28 \\
\hline 5 & Декан & 6 & 40 & 39 & 42 & 33 \\
\hline 6 & Этанол & 11 & 41 & 27 & 36 & 2 \\
\hline 7 & Метилэтилкетон & 4 & 36 & 29 & 39 & 16 \\
\hline 8 & Нитрометан & 5 & 37 & 37 & 41 & 19 \\
\hline 9 & Бензол & 0,3 & 34 & 31 & 40 & 16 \\
\hline 10 & Пиридин & 1 & 8 & 37 & 32 & 24 \\
\hline 11 & & & & & & \\
\hline
\end{tabular}

Для акцептора электронов, нитрометана характер приведенной картины изменяется, и трифенильные производные элементов пятой группы Периодической системы располагаются в следующем ряду: $\mathrm{Sb} \quad\left(-\Delta \mathrm{H}^{\circ}=41 \quad\right.$ кДж/моль $)>\mathrm{As}$ $\left(-\Delta \mathrm{H}^{\mathrm{o}}=37 \quad\right.$ кДж/моль $)>\mathrm{P} \quad\left(-\Delta \mathrm{H}^{\circ}=37 \quad\right.$ кДж/моль $)>\mathrm{Bi} \quad\left(-\Delta \mathrm{H}^{\circ}=19 \quad\right.$ кДж/моль $)>\mathrm{N}$ $\left(-\Delta \mathrm{H}^{\mathrm{o}}=5\right.$ кДж/моль). Сорбция метилэтилкетона, которая в условиях газожидкостной хроматографии описывается ориентационным взаимодействием метилэтилкетона со структурными фрагментами молекулы сорбента, характеризуется более высокими значениями для сурьмы $\left(-\Delta \mathrm{H}^{0}=39\right.$ кДж/моль) с уменьшением в сторону фосфора $\left(-\Delta \mathrm{H}^{\mathrm{o}}=36\right.$ кДж/моль), мышьяка $\left(-\Delta \mathrm{H}^{0}=29\right.$ кДж/моль $)$, висмута $\left(-\Delta \mathrm{H}^{\mathrm{o}}=16\right.$ кДж/моль $)$ и азота $\left(-\Delta \mathrm{H}^{\mathrm{o}}=4\right.$ кДж/моль). Энтальпия сорбции н-алканов возрастает с увеличением числа атомов углерода в их молекуле практически для всех трифенильных производных. При этом для азота она имеет низкие значения и практически не зависит от количества атомов углерода в молекуле алкана.

С целью оценки вклада в относительную электронодонорную способность соединений элементов пятой группы Периодической системы различных по природе межмолекулярных взаимодействий, проводился сравнительный анализ хроматографических факторов полярности указанных соединений на основе пространственных представлений. На рис. 2 приведена развертка на плоскости $\mathrm{F}_{\mathrm{z}}, \mathrm{F}_{\mathrm{x}}, \mathrm{F}_{\mathrm{s}}, \mathrm{F}_{\mathrm{y}}$ правильного тетраэдра для трифенильных производных. Из рис.2 видно, что в развертке на плоскости $\mathrm{F}_{\mathrm{z}}, \mathrm{F}_{\mathrm{x}}, \mathrm{F}_{\mathrm{s}}, \mathrm{F}_{\mathrm{y}}$ правильного тетраэдра экспериментальные точки, соответствующие трифенильным производным, группируются в различных областях. Последовательность расположения экспериментальных точек практически соответствует по- 
рядку возрастания величины заряда ядра центрального атомы от азота к висмуту. При этом вклад $\pi$-донорного взаимодействия (бензол) равноценен для всех изученных соединений, т.е. не зависит от свойств центрального атома, и, очевидно, определяется только межмолекулярным взаимодействием с фенильными кольцами и стерической доступностью к взаимодействию в системе сорбат - сорбент.

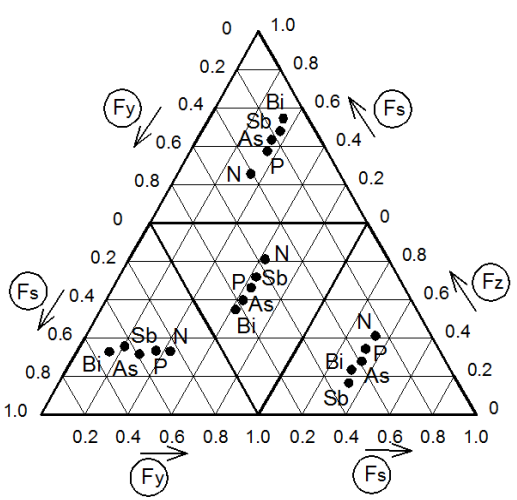

Рис.2. Развертка на плоскости $\left(\mathrm{F}_{\mathrm{z}}\right)$, $\left(\mathrm{F}_{\mathrm{x}}\right),\left(\mathrm{F}_{\mathrm{s}}\right),\left(\mathrm{F}_{\mathrm{y}}\right)$ правильного тетраэдра для трифенильных производных элементов пятой группы Периодической системы. Доли рассчитаны из логарифмических индексов удерживания стандартных сорбатов: метилэтилкетона $\left(\mathrm{F}_{\mathrm{z}}\right)$, бензола $\left(\mathrm{F}_{\mathrm{x}}\right)$, пиридина $\left(\mathrm{F}_{\mathrm{s}}\right)$, этанола $\left(\mathrm{F}_{\mathrm{y}}\right)$.

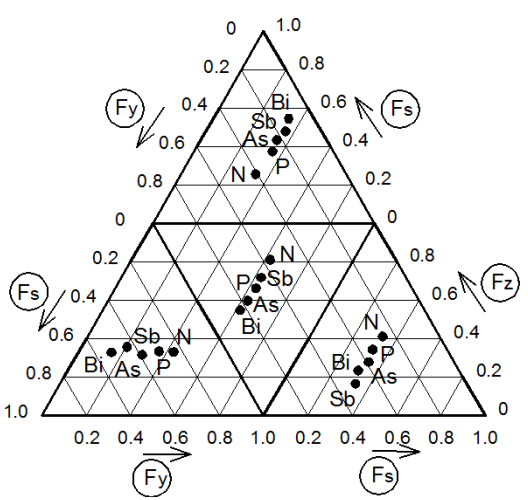

Рис.3. Зависимость хроматографических факторов полярности (у) от (х) для трифенильных производных элементов пятой группы Периодической системы (проекция на плоскость (х-у)

из пятимерного пространства).

На рис. 3 приведена зависимость хроматографических факторов полярности (у), которые определяются по этанолу, от хроматографического фактора полярности (х)- бензол, который характеризует проекцию на плоскость (у-х) из пятимерного пространства по аналогии с работой [22], где описана методика оценки сорбентов в многомерном пространстве. Как видно из рис.3, все исследуемые вещества характеризуются относительно высокой способностью к образованию межмолекулярной водородной связи. В этом случае трифенильные производные элементов пятой группы Периодической системы располагаются в разных областях многомерной фигуры и характеризуют диапазон селективных возможностей исследуемого ряда соединений.

\section{Заключение}

В результате оценки электронодонорной способности элементов пятой группы Периодической системы методом газовой хроматографии на насадочных хроматографических колонках показано влияние центрального атома молекул трифенильных производных на их сорбционные свойства по отношению к органическим веществам различной физико-химической природы, которое определяется межмолекулярным взаимодействием в системе сорбат-сорбент. Результаты могут быть использованы при синтезе новых сорбентов для газо-жидкостной хроматографии с регулируемой селективностью разделения за счет введения в структуру молекулы неподвижной жидкой фазы различных функциональных групп, способных к межмолекулярным взаимодействиям. 


\section{Список литературы}

1.Танеева А.В., Карташова А.А., Федоренко О.И., Макаров А.Н., Новиков В.Ф. Патент РФ.№ 2496572, 2013.

2. Танеева А.В., Карташова А.А., Левин И.С., Новиков В.Ф. Патент РФ, № 2446008, 2012.

3. Новиков В.Ф. // Журнал физической химии. 1993. Т.67. №4. С.848-853.

4. Танеева А.В., Ильина О.В., Левин И.С., Карташова А.А. и др. // Вестник Казанского технологического университета. 2011. №3. C.286-289.

5. Халитов К.Ф., Танеева А.В., Карташова А.А., Новиков В.Ф. // Известия высших учебных заведений. Серия: Химия и химическая технология. 2011. №11. С.57-60.

6. Карташова А.А., Новиков В.Ф. // Известия высших учебных заведений. Проблемь энергетики. 2016. №1-2. С.47-52.

7. Гурьянова Е.Н., Соболев А.Н., Ромм И.П., Бельский В.К. Структурная химия триарилпроизводных 5 группы. В кн.: Химическая связь и строение молекул. М. Наука. 1984. С. 97-107.

8. Эпштейн Л.М., Новикова 3.С., Ашкинадзе Л.Д., Рабичева С.О. и др. // Известия $А H$ СССР, сер. хим. 1974. №1. С. 65-71.

9. Халитов К.Ф., Новиков В.Ф., Халитов Ф.Р. // Журнал общей химии. 2016. Т.86. №10. C.1640-1646.

10. Гамаюрова В.С. // Успехи химии. 1981. T.50. C.1601-1626.

\section{References}

1. Taneeva A.V., Kartashova A.A., Fedorenko O.I., Makarov A.N. et al., Patent RF, No $2496572,2013$.

2. Taneeva A.V., Kartashova A.A., Levin I.S., Novikov V.F., Patent RF, No 2446008, 2012.

3. Novikov V.F., J. of Physical Chemistry, 1993, Vol. 67, No 4, pp. 848-853.

4. Taneeva A.V., Ilyin O.V., Levin I.S., Kartashova A.A., et al., Vestnik Kazanskogo tehnologicheskogo universiteta, 2011, No 3, pp. 286289.

5. Halitov K.F., Taneeva A.V., Kartashova A.A., Novikov V.F., Izvestija vysshih uchebnyh zavedenij. Serija: Himija i himicheskaja tehnologija, 2011, No 11, pp. 57-60.
11. Пурдела Д., Вылчану Р. Химия органических соединений фосфора. М. Химия. 1972. $230 \mathrm{c}$.

12. Хроматография. Основные понятия. Терминология. Сборник научно-нормативной терминологии. 1997. Вып. 114. 29 с.

13. ГОСТ 17567-81. Термины и определения в газовой хроматографии.

14. Ettre I.S. // Appl. Chem. 1993. Vol. 65. pp. 819-872.

15. Kovatc E. Helv. Chim. Acta. 1958. Vol. 41, pp. 1615-1918.

16. Rohrshneider L.R.// Z.Analyt.Chem. 1959. Vol.170. No 1, pp. 257-263.

17. Rohrshneider L.R// J. G. Chromatogr. 1966. Vol.22. No 1. pp. 6-22.

18. Айвазов Б.В. Введение в хроматографию: Учебн. пособие для хим. спец. вузов. М. Высшая школа. 1983. 240 с.

19. Синкевич А.В.,Новиков В.Ф., Танеева А.В. // Известия Казанского государственного архитектурно-строительного универcumema. 2009. №1(11). C. 292-296.

20. Фадеев Г.Н. Пятая вертикаль Периодической системы. М. Просвещение.1979. 192 c.

21. Реутов О.А. Теоретические основы органической химии. М. МГУ. 1964. 230с.

22. Vigdergaus M.S., Bankovskaya T.R. // Chromatographia. 1976. Vol. 9. pp. 548-553.

6. Kartashova A.A., Novikov V.F., Izvestiya vysshikh uchebnykh zavedenii. Problemy energetiki, 2016, No 1-2, pp. 47-52.

7. Gur'janova E.N., Sobolev A.N., Romm I.P., Bel'skij V.K. Strukturnaja himija triarilproizvodnyh 5 gruppy. $\mathrm{V}$ kn.: Himicheskaja svjaz' i stroenie molekul. M., Nauka Publ., 1984, pp. 97-107.

8. Jepshtejn L.M., Novikova Z.S., Ashkinadze L.D., Rabicheva S.O. et al., Izvestija AN SSSR, ser. him., 1974, No 1, pp. 65-71.

9. Halitov K.F., Novikov V.F., Halitov F.R., J. Of General Chemistry, 2016, Vol. 86, No 10, pp.1640-1646.

10.Gamajurova V.S., Uspehi khimii, 1981, Vol. 50, pp.1601-1626. 
11.Purdela D., Vylchanu R. Chemistry of organic phosphorus compounds, M., Chemistry Publ., 1972, 230 p.

12.Chromatography. Basic concept. Terminology. Collection of scientific and normative terminology, 1997, Iss. 114, 29 p.

13.GOST 17567-81. Terms and definitions in gas chromatography.

14.Ettre I.S., Appl. Chem, 1993, Vol. 65, pp. 819-872.

15.Kovatc E., Helv. Chim. Acta, 1958, Vol. 41, pp. 1615-1918.

16.Rohrshneider L.R., Z. Analyt. Chem., 1959, Vol.170, No 1, pp.257-263.

17.Rohrshneider L.R., J. G. Chromatogr., 1966, Vol.22, No 1, pp.6-22.

Танеева Алина Вячеславовна - доцент, кандидат химических наук, Казанский государственный энергетический университет, кафедра Энергообеспечение предприятий и энергоресурсосберегающих технологий, Казань

Новиков Вячеслав Федорович - профессор, доктор химических наук, Казанский государственный энергетический университет, кафедра Энергообеспечение предприятий и энергоресурсосберегающих технологий, Казань

Дмитриева Александра В. - аспирант, Казанский государственный энергетический университет, кафедра Энергообеспечениея предприятий и энергоресурсосберегающих технологий, Казань

Ву Нгок Зан - аспирант, Казанский государственный энергетический университет, кафедра Энергообеспечение предприятий и энергоресурсосберегающих технологий, Казань

Нгуен Зуи Хынг - аспирант, Казанский государственный энергетический университет, кафедра Энергообеспечение предприятий и энергоресурсосберегающих технологий, Казань
18. Ajvazov B.V. Vvedenie v khromatografiju: Uchebn. posobie dlja him. spec. vuzov, M., Vysshaja shkola, 1983, $240 \mathrm{p}$.

19.Novikov V.F., Sinkevich A.V., Taneeva A.V. Izvestija Kazanskogo gosudarstvennogo ahchiteckturno-stroyitelnogo universiteta , 2009, No 1(11), pp. 292-296.

20. Fadeev G.N. Pjataja vertikal' Periodicheskoj sistemy, M., Prosveschenie Publ., 1979, $192 \mathrm{p}$.

21. Reutov O.A. Teoreticheskie osnovy organicheskoj khimii, M., MGU Publ., 1964, 230p.

22.Vigdergaus M.S., Bankovskaya T.R., Chromatographia, 1976, Vol. 9, pp.548-553.

Taneeva Alina V. - associate Professor, candidate of chemical Sciences, Kazan state power engineering University, Department of energy supply of enterprises and energy-saving technologies, Kazan, e-mail: alinataneeva@mail.ru

Novikov Vyacheslav F. - Professor, doctor of chemical Sciences, Kazan state power engineering University, Department of energy supply of enterprises and energy-saving technologies, Kazan

Dmitrieva Alexandra V. - graduate student, Kazan state power engineering University, Department of energy supply of enterprises and energysaving technologies, Kazan

Wu Ngok Zan - graduate student, Kazan state power engineering University, Department of energy supply of enterprises and energy-saving technologies, Kazan

Nguyen Zuy Hung - graduate student, Kazan state power engineering University, Department of energy supply of enterprises and energy-saving technologies, Kazan 